\title{
Supernova Remnants as Clues to Their Progenitors
}

\author{
Daniel Patnaude and Carles Badenes
}

\begin{abstract}
Supernovae shape the interstellar medium, chemically enrich their host galaxies, and generate powerful interstellar shocks that drive future generations of star formation. The shock produced by a supernova event acts as a type of time machine, probing the mass loss history of the progenitor system back to ages of $\sim 10$ 000 years before the explosion, whereas supernova remnants probe a much earlier stage of stellar evolution, interacting with material expelled during the progenitor's much earlier evolution. In this chapter we will review how observations of supernova remnants allow us to infer fundamental properties of the progenitor system. We will provide detailed examples of how bulk characteristics of a remnant, such as its chemical composition and dynamics, allow us to infer properties of the progenitor evolution. In the latter half of this chapter, we will show how this exercise may be extended from individual objects to SNR as classes of objects, and how there are clear bifurcations in the dynamics and spectral characteristics of core collapse and thermonuclear supernova remnants. We will finish the chapter by touching on recent advances in the modeling of massive stars, and the implications for observable properties of supernovae and their remnants.
\end{abstract}

\section{Introduction}

Supernova remnants provide a unique view of the supernova (SN) phenomenon. The hundreds of extragalactic supernovae $(\mathrm{SNe})$ discovered each year by modern

Daniel Patnaude

Smithsonian Astrophysical Observatory, 60 Garden St, Cambridge, MA 02138, e-mail: dpatnaudedefa.harvard.edu

Carles Badenes

Department of Physics and Astronomy, and Pittsburgh Particle Physics, Astrophysics, and Cosmology Center (PITT-PACC), University of Pittsburgh, 3931 O'Hara St, Pittsburgh, PA 15260 e-mail:

badenesepitt.edu 
surveys are unresolved point sources which fade before they can interact with any circumstellar material outside of their most immediate surroundings. Before they fade, they probe the evolution of the progenitor on timescales of $\sim 100-1000$ years before the explosion. This is because the typical outflow speeds from the progenitor are $\sim 10-1000 \mathrm{~km} \mathrm{~s}^{-1}, 100-1000 \times$ slower than the typical supernova blastwave velocity.

In contrast, supernova remnants (SNRs) in the Local Group galaxies can be resolved in some detail as they interact with the bulk of the circumstellar material expelled by their progenitors during pre-SN mass loss over much longer timescales. Additionally, SNRs are surrounded by resolved stellar populations that spawned their SN progenitors (Badenes et al., 2009, Williams et al., 2014, Jennings et al. 2014), and they have become prime hunting grounds in the search for their surviving binary companions (e.g., Edwards et al., 2012, Kerzendorf et al., 2014). X-ray spectra of SNRs can reveal emission from neutron-rich isotopes in the ejecta which are sensitive probes of SN explosion mechanisms (e.g., Yamaguchi et al., 2015). In short, SNR observations can constrain aspects of SNe and their progenitors that are either difficult or impossible to access by optical SN observations.

In this chapter, we review the clues that supernova remnants provide about their progenitor's evolution and ultimate fate. We begin by highlighting studies of both Type Ia and core-collapse SNRs which inferred properties of their progenitors and evolution. We then discuss recent techniques that use bulk SNR observables to constrain SN progenitor properties. We conclude by touching on recent results in massive star evolution which may be relevant towards understanding observable properties in supernova remnants. For further background and a different perspective of these topics, see the reviews by Badenes (2010) and Vink (2012).

\section{Supernova Remnant - Progenitor Connections}

The main challenge associated with studying supernova remnants is related to their type. Classically, SNe have been typed by their optical spectrum around maximum light (Filippenko, 1997). In general terms, Type Ia supernovae show no hydrogen in their optical spectra, with Type Ia (thermonuclear) SNe exhibiting strong Si II absorption around $615 \mathrm{~nm}$. Type $\mathrm{Ib} / \mathrm{c}$ are core-collapse supernovae, and differ from Ia SNe in that they possess either a weak or non-existent silicon absorption feature, and little (Type Ib) or no (Ic) absorption from He I. The amount of helium in the optical spectrum may provide insight into the degree of mass-loss in the progenitor prior to core-collapse. In contrast, Type II SNe do show hydrogen in their spectra, and are further subdivided into types IIP, IIL, IIb and IIn, based on the evolution and characteristics of their light curve or, in the case of Type IIn SNe, their optical spectrum.

While SNe are common and somewhat accessible to typing, SNRs are evolved objects, and a priori it is non-trivial to associate them with each of the established SN types, though the detection of optical light echoes from the SN event do provide 
clues in some cases (Rest et al. 2008). However, given the wealth of data available, a challenging opportunity exists to connect remnant properties back to the properties of the progenitor.

Connecting remnant properties to those of the progenitor often involves a detailed analysis of the remnant's broadband spectrum, combined with comparisons to hydrodynamical models for supernova remnant ejecta evolution. In some cases such as those discussed below, this has produced interesting constraints. However, bulk properties such as emission line strengths, energy centroids, and chemical abundances depend on different aspects of a SN explosion than those of optical SNe. These properties can act as crucial benchmarks for specific stellar evolution scenarios. In $\S 3$ of this chapter, we explore how these so called "bulk observables" connect directly to the evolution of the progenitor, and how theorists and observers are connecting detailed stellar evolution models to properties of young supernova remnants.

When studying SNRs, both as individual objects and as a class, the main properties to consider are the chemical composition of the ejecta and the dynamics of the blast wave. The spectrum of a SNR in the X-ray band is a combination of nonthermal emission from the blast wave, and thermal emission from both swept-up circumstellar material and shocked supernova ejecta. The chemical composition of the shocked ejecta is determined by fitting models to the observed X-ray spectrum, which often requires some knowledge of the SNR dynamics (Borkowski et al. 2001). In turn, the blast wave dynamics are inferred by relying on critical assumptions about the relationship between the shock speed and the measured electron temperature. Only in certain cases, such as in the Galactic SNR Cas A, are the dynamics directly observed (DeLaney \& Rudnick, 2003, Patnaude \& Fesen, 2009).

In most cases, the morphology is compared to self-similar models for blast wave evolution, which again require assumptions about the remant's age, energetics, and circumstellar environment. These methods are vulnerable to circular logic and can result in detailed statements about the properties of a given SNR that fail to properly account for the considerable uncertainties introduced by the models. In other words, the critical assumptions made in connecting spectral properties to dynamics often compromise the the connections made back to the progenitor evolution.

As an example, specific stellar evolution scenarios from SN Ia progenitors predict circumstellar structures that range from the $\rho \propto r^{-2}$ profiles that are the signature of isotropic mass-loss to relatively large low-density energy-driven cavities excavated by fast outflows from accreting white dwarfs (Badenes et al., 2007; Shen et al., 2013). As seen in Figure 1, the prompt radio and X-ray emission from $\mathrm{SNe}$ (Chomiuk et al. 2016; Margutti et al. 2014), weeks or months after the explosion, can only probe circumstellar material (CSM) structures up to a few hundred AU, composed of material expelled by the progenitor hundreds to thousands of years before the explosion, depending on the outflow velocity.

In contrast, SNRs that are hundreds or thousands of years old can probe CSM structures up to several pc in size, expelled by the progenitor several thousands to millions of years before the explosion. These spatial scales are representative of typical stellar wind blown bubbles (Koo \& McKee, 1992), and the associated 
temporal scales are directly relevant to stellar evolution scenarios for Type Ia SN progenitors (Wang \& Han, 2012). For CC SNRs, the argument is even stronger, as mass-loss is often the single most uncertain ingredient in stellar evolution models for SN progenitors (Smith, 2014).

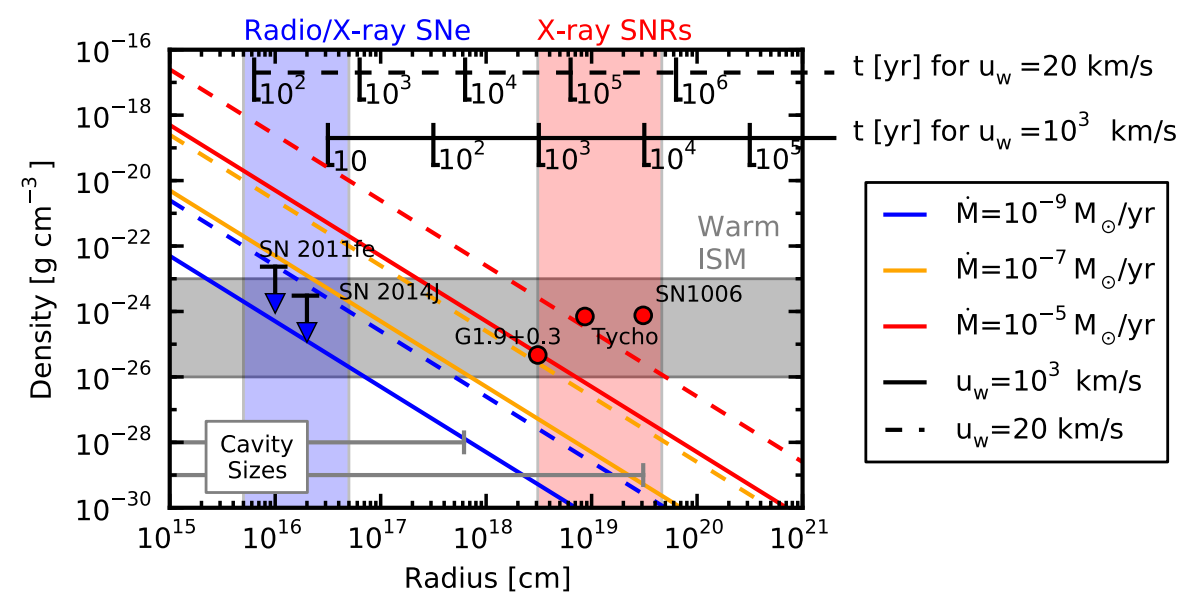

Fig. 1 Schematic circumstellar density profiles for isotropic SN Ia progenitor outflows (colored plots, dashed for $\mathrm{u}_{\mathrm{w}}=20 \mathrm{~km} \mathrm{~s}^{-1}$; solid for $\mathrm{u}_{\mathrm{w}}=10^{3} \mathrm{~km} \mathrm{~s}^{-1}$ ), compared to the typical densities in the warm phase of the interstellar medium (horizontal grey band) (Ferrière 2001). The rulers on top of the plots show the time before the explosion (in yr) when the material at that radius was ejected by the SN progenitor. The rulers on the bottom show the typical sizes of circumstellar cavities excavated by fast outflows (Badenes et al. 2007, Shen et al. 2013). The radii probed by X-ray/radio follow-up observations of SNe and studies of Type Ia SNRs are shown by blue and red shaded vertical stripes. The blue arrows and red circles show upper limits for two Type Ia SNe (Chomiuk et al. 2016, Pérez-Torres et al. 2014) and measurements for three Type Ia SNRs (Raymond et al. [2007, Slane et al. [2014, Borkowski et al. 2014).

A different approach is instead of studying the minute details of individual objects, one probes the general characteristics of SNRs to see how they relate to the expected characteristics of a model derived from a potential progenitor scenario. This approach has been successful in Type Ia SNRs, and is now beginning to be explored in core collapse scenarios.

\subsection{Type Ia SNR - Progenitor Connections}

Despite decades of efforts, the progenitors of Type Ia SNe remain unidentified. The absence of hydrogen and helium in the optical spectra of SN Ia, their association with both young and old stellar populations, the absence of a detectable shock breakout in the early light curves, and the chemical composition of the ejecta, as inferred from optical and X-ray observations, imply that the exploding star is a 
carbon-oxygen (C/O) white dwarf (WD). The exact events leading to the explosion are unclear, but after the degenerate matter is somehow ignited, a thermonuclear runaway consumes most of the star, synthesizing $0.5-1.0 \mathrm{M}_{\odot}$ of ${ }^{56} \mathrm{Ni}$, which powers the optical lightcurve through the decay chain ${ }^{56} \mathrm{Ni} \rightarrow{ }^{56} \mathrm{Co} \rightarrow{ }^{56} \mathrm{Fe}$.

Different SN Ia progenitor scenarios attempt to provide a physical explanation for the destabilization of the WD and the subsequent explosion. In the single-degenerate (SD) scenario, the WD grows slowly in mass by accretion from a non-degenerate companion, until it gets close to the Chandrasekhar limit $\left(\mathrm{M}_{\mathrm{Ch}} \approx 1.4 \mathrm{M}_{\odot}\right)$. In the double degenerate (DD) scenario, the WD explodes after a dynamical merger or a collision with another WD, forming a short-lived object whose mass does not necessarily have to be $\mathrm{M}_{\mathrm{Ch}}$. See Hillebrandt et al. (2013) and Maoz et al. (2014) for recent reviews on SN Ia progenitors.

The so-called 'SN Ia progenitor problem' persists because even though there is a great deal of indirect evidence supporting both scenarios, a definite 'smoking gun' remains elusive (see Maoz et al. 2014, for a discussion). In the SD scenario, the WD accretes mass at a rate of $\dot{M}=\sim 10^{-7} \mathrm{M}_{\odot} \mathrm{yr}^{-1}$ (Shen \& Bildsten 2007). At these rates, stable hydrogen burning can occur on the surface of the WD, which allows steady mass growth to $\mathrm{M}_{\mathrm{Ch}}$. Accretion at higher rates can lead to the expansion of the accretor and engulfment of the companion into a common envelope phase, which effectively halts the growth of the WD. Solutions to this problem have been proposed, however, in the form of fast optically thick accretion winds that regulate the accretion (Hachisu et al., 1996). Lower accretion rates can result in the accumulation of degenerate material on the WD, which will eventually ignite as a nova where much of the accreted material and potentially some of the accretor mass is ejected, effectively eroding the mass of the WD.

In the DD scenario, a more massive white dwarf merges or collides with a lowermass WD and this rapid episode of mass accretion can effectively avoid problems related to inefficient mass growth in the SD scenario, and result in a SN Ia explosion, not necessarily close to $\mathrm{M}_{\mathrm{Ch}}$ (van Kerkwijk et al. 2010). However, offcenter ignition in the primary WD can result in the formation of a O/Ne WD and an accretion-induced collapse to a neutron star without a SN Ia explosion (Saio \& Nomoto, 1985).

Thus two key observational probes of the SN Ia progenitor problem are the total mass of the ejecta (i.e., whether it is close to $\mathrm{M}_{\mathrm{Ch}}$ or not) and the presence or absence of a fast accretion wind outflow before the explosion. SNR studies can effectively constrain both ejecta mass and pre-explosion outflows.

When a WD explodes close to $\mathrm{M}_{\mathrm{Ch}}$ in the SD scenario, it will have a dense $\left(\rho \gtrsim 2 \times 10^{8} \mathrm{~g} \mathrm{~cm}^{-3}\right)$ core where efficient electron captures can take place. This results in a significant production of neutron-rich isotopes such as ${ }^{58} \mathrm{Ni}$ and ${ }^{55} \mathrm{Mn}$ in the innermost $\sim 0.2 \mathrm{M}_{\odot}$ of the WD (Brachwitz et al., 2000). In contrast, a SN Ia explosion with a DD progenitor will not present this neutronized core, unless it happened exactly at $\mathrm{M}_{\mathrm{Ch}}$.

X-ray observations of Type Ia SNR can reveal the presence or absence of this neutronized core by comparing diagnostic mass ratios like $\mathrm{Ni} / \mathrm{Fe}$ and $\mathrm{Mn} / \mathrm{Fe}$ to the predictions of both $\mathrm{M}_{\mathrm{Ch}}$ and sub- $\mathrm{M}_{\mathrm{Ch}}$ models (Park et al. 2013). In the evolved 
Galactic SNR 3C 397, Yamaguchi et al. (2015) found high ratios of Ni/Fe and Mn/Fe in the X-ray spectrum. As seen in Figure 2, the $\mathrm{Ni} / \mathrm{Fe}$ and $\mathrm{Mn} / \mathrm{Fe}$ ratios are among the highest reported for Type Ia SNRs, and strongly indicate that the progenitor must have exploded close to $\mathrm{M}_{\mathrm{Ch}}$, which is more naturally explained by the SD scenario.
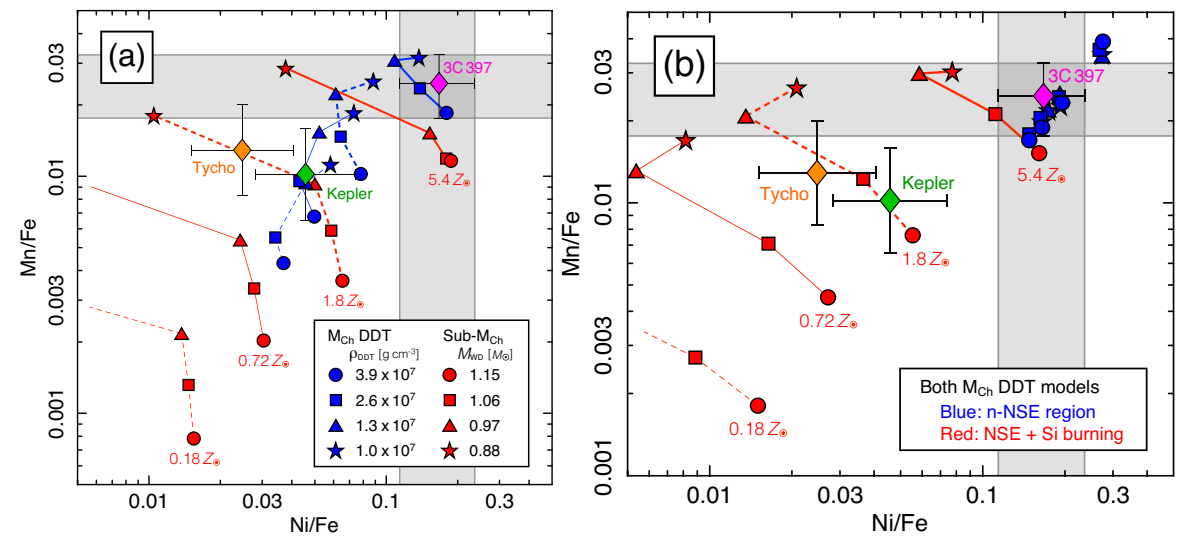

Fig. 2 (a) $\mathrm{Ni} / \mathrm{Fe}$ and $\mathrm{Mn} / \mathrm{Fe}$ mass ratios in SN Ia models, compared with the observed values in 3C 397 (magenta diamond and gray regions). Blue symbols represent $M_{\mathrm{Ch}}$ delayed-detonation models with different values of $\rho_{\mathrm{DDT}}$ as indicated in the legend. Red symbols represent sub- $M_{\mathrm{Ch}}$ detonation models with WD masses given in the legend. Models with the same progenitor metallicity are connected by lines: $5.4 Z_{\odot}$ (thick solid), $1.8 Z_{\odot}$ (thick dashed), $0.72 Z_{\odot}$ (thin solid), and $0.18 Z_{\odot}$ (thin dashed), The values for Kepler and Tycho indicated by the green and orange diamonds are calculated using the line fluxes from Park et al. (2013) and Yamaguchi et al. (2014). (b) Same as panel (a), but the values predicted for the innermost $0.2 \mathrm{M}_{\odot}$ that is dominated by the n-NSE regime (blue) and the other regions (red) from the $M_{\mathrm{Ch}}$ models are separately shown. The observed mass ratios for 3C 397 can be well explained by the nucleosynthesis that occurs in the n-NSE regions of standard metallicity models. (Figures reproduced with the permission of the authors; Yamaguchi et al. 2015. Figure 4, pp. 5).

A key prediction of the SD scenario is the presence of circumstellar material (CSM). One possible source of this CSM are the fast, optically thick winds that are required to stabilize accretion and allow the WD to grow to $\mathrm{M}_{\mathrm{Ch}}$ and avoid a common-envelope phase (Hachisu et al. 1996). These fast winds have mass loss rates comparable to the accretion rate $\left(1-5 \times 10^{-7} \mathrm{M}_{\odot} \mathrm{yr}^{-1}\right)$, and velocities larger than the escape velocity from the surface of a massive WD (several hundred $\mathrm{km} \mathrm{s}$ ${ }^{-1}$ ), resulting in large mechanical luminosities.

By the time of the SN explosion, these outflows should have carved out large (several pc in diameter) low-density cavities around the progenitor system. Even if accretion winds are not active, it is hard to envision any realization of the SD scenario that does not lead to some kind of CSM, because the accretion process cannot be $100 \%$ efficient. For outflow velocities slower than those found in accretion winds, the CSM should stay close to the progenitor, and geometric dilution will lead to a density profile of the form $\rho_{\mathrm{CSM}} \sim A r^{-2}$, where $A$ is a constant of proportionality 
dependent upon the mass-loss rate and wind velocity. Badenes et al. (2007) studied the impact of several CSM distributions on SNR evolution, and concluded that most Type Ia SNRs show no significant evidence for the presence of CSM.

In Figure 3 we show an updated comparison between the models of Badenes et al. (2007) and the $\mathrm{Fe} \mathrm{K} \alpha$ centroids measured for Type Ia SNRs by Yamaguchi et al. (2014) (see Section 3 below for a more detailed explanation of these measurements). The physical radii and the ionization state of $\mathrm{Fe}$ in Type Ia SNRs are in good agreement with SNR models where Type Ia SN ejecta interact with an undisturbed ambient medium (shaded regions). Models with a $\rho_{\mathrm{CSM}} \sim A r^{-2}$ circumstellar profile (blue plot) lead to small, highly ionized SNRs that do not appear in the observed sample (though many core-collapse SNRs fit this description, see Section 3). Models expanding into low density cavities excavated by accretion winds lead to large, low-ionization SNRs. Although these objects are not common, there is at least one example in the sample from Yamaguchi et al. (2014): SNR RCW 86. This SNR has long been suspected to be a cavity explosion, and its bulk dynamics are hard to interpret without a fast, sustained outflow from the progenitor, with properties similar to those of accretion winds (Badenes et al., 2007, Williams et al., 2011).

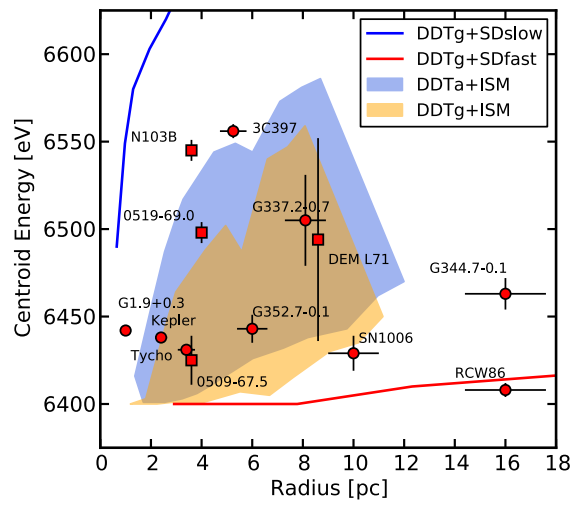

Fig. 3 Fe K $\alpha$ line centroid as a function of radius for the Type Ia SNRs in Yamaguchi et al. 2014, plus DEM L71 (Maggi et al. 2016). The shaded regions represent theoretical predictions from Type Ia SNR models interacting with a uniform interstellar medium (Badenes et al. 2003 2005): orange for the ${ }^{56} \mathrm{Ni}$-poor delayed detonation model DDTg, blue for bright ${ }^{56} \mathrm{Ni}$-rich delayed detonation model DDTa. The colored lines represent theoretical predictions from Badenes et al. (2007) for model DDTg interacting with two circumstellar profiles calculated assuming an SD progenitor, red for an accretion wind outflow with a large cavity (SDfast) and blue for a $\rho \propto r^{-2}$ outflow (SDslow).

A curious feature of Figure 3 is the agreement shown between Kepler's SNR and models for an unmodified ambient medium. Shown in Figure 4 (left) is an X-ray image of Kepler, showing clear signs of circumstellar interaction. Patnaude et al. (2012) modeled the dynamics and X-ray emission for a Type Ia model expanding in the dense wind of an AGB companion (Chiotellis et al. 2012). Patnaude et al. (2012) jointly modeled the blastwave dynamics and X-ray spectrum of the SNR. The line 
energy centroids of prominent emission lines of silicon and iron are sensitive to the circumstellar environment the remnant is expanding into. Patnaude et al. (2012) found that in order to match both the dynamics and bulk X-ray spectral properties, a small cavity, of order $0.1 \mathrm{pc}$ in radius, is required around the progenitor. They suggest that the cavity could have been formed just prior to thermonuclear runaway, as the accretion wind pushes out against the dense wind from the AGB companion.

Given the high Fe content observed in the spectrum of Kepler's SNR (Patnaude et al. 2012; Katsuda et al. 2015a), there is little doubt that it is the result of a Ia and the CSM wind scenario argued by Chiotellis et al. (2012); Patnaude et al. (2012); Katsuda et al. (2015a) seems to favor the SD channel, though fast accretion winds appear ruled out. However, Kerzendorf et al. (2014) obtained spectra for 24 stars with $L>10 L_{\odot}$, and none showed evidence for being a former donor star, including no red giant, AGB, or post-AGB surviving companions in the inner $40^{\prime \prime}$ of the remnant. Similar searches for companions in the LMC remnants SNR 0519-67.5 and 0509-67.5 also turn up no evidence for a surviving companion (Schaefer \& Pagnotta, 2012, Edwards et al. 2012). While this isn't firm proof for the viability of the DD scenario, it does suggest that, at least in these cases, the double degenerate progenitor model could be required in order to produce these SNRs.

To conclude, observations of Type Ia SNRs provide clear evidence for a $\mathrm{M}_{\mathrm{Ch}}$ SN Ia progenitor in SNR 3C 397 (Yamaguchi et al., 2015), and the presence of a fast, sustained outflow in SNR RCW 86 (Badenes et al., 2007; Williams et al., 2011). Taken at face value, this seems to support the SD scenario for at least some local Type Ia SNe. However, the bulk properties of most Type Ia SNRs are at odds with the CSM configurations expected in SD progenitors (see Figure 3 and Badenes et al. 2007, for a detailed discussion). More contrived CSM profiles are certainly possible in some cases, as shown by Patnaude et al. (2012) for Kepler, but a more likely explanation is that DD progenitors also make a significant contribution to the local SN Ia rate (see Badenes \& Maoz, 2012). A mixture of progenitor channels has interesting implications for cosmology (Ponder et al., 2016).

\subsection{Core Collapse SNRs - Progenitor Connections}

A good review concerning the connections between young supernova remnants and their progenitors can be found in Chevalier (2005). They note that different supernova types arise form different zero age main sequence masses, with $\mathrm{SNe}$ IIP occuring in the 8-15 $\mathrm{M}_{\odot}$ range, $\mathrm{SNe} \mathrm{Ib} / \mathrm{c}$ occuring in stars with ZAMS mass $\gtrsim 35$ $\mathrm{M}_{\odot}$, and IIb/IIL SNe resulting from stars in the middle, with observational results providing information on the progenitor to supernova type relationship Smartt(e.g., 2015).

If firm connections between progenitor and supernova type can be established, then connecting a remnant to it's parent supernova provides an indirect path back to the progenitor type. Chevalier (2005) attempted this for several objects, including the Crab, 3C 58, and G292.0+1.8. In the Crab, measured abundances suggest that the 

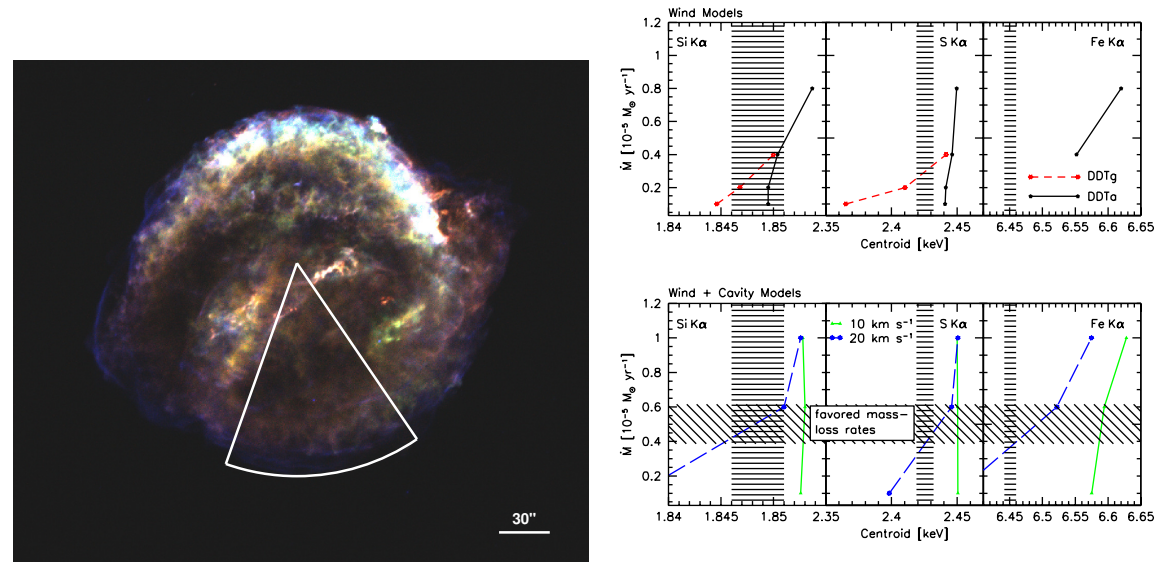

Fig. 4 Left: Kepler's SNR viewed in X-rays with Chandra ACIS-S3. The RGB image shows 0.4$0.75 \mathrm{keV}$ emission in red, $0.75-1.2 \mathrm{keV}$ emission in green, and $1.2-7.0 \mathrm{keV}$ emission in blue. In the image, north is up and east is to the left. The outlined region corresponds to the blastwave-ejecta interaction discussed in Patnaude et al. (2012). Right: In the upper panel, we plot the measured versus computed line centroids for $\mathrm{Si} \mathrm{K} \alpha, \mathrm{S} \mathrm{K} \alpha$, and $\mathrm{Fe} \mathrm{K} \alpha$, for the DDTa (black solid) and DDTg (red dashed) models. The hatched region in each panel corresponds to the measured centroid including the $90 \%$ confidence interval The DDTg models and a subset of the DDTa models $(\dot{M}<$ $4 \times 10^{-6} \mathrm{M}_{\odot} \mathrm{yr}^{-1}$ ) do not produce any $\mathrm{Fe} \mathrm{K}$ emission. In the lower panel, we plot the line centroids for the DDTa cavity models for $\mathrm{v}_{\text {wind }}=10$ (green solid) and 20 (blue dashed) $\mathrm{km} \mathrm{s}^{-1}$, for a range of mass-loss rates. The line centroids and errors are indicated by the vertical hatched regions in each panel. The allowed mass-loss rates as dictated by the comparison between the measured and modeled line centroids are marked by the horizontal cross hatched region (Figures reproduced with the permission of the authors; Patnaude et al. 2012).

progenitor lacked an O-rich mantle, placing the progenitor star at the low end of the supernova progenitor mass range, $\sim 8-10 \mathrm{M}_{\odot}$, suggesting that the Crab supernova was of the Type IIP variety. On the other hand, the SNR G292.0+1.8 probably arises from a more massive progenitor, as the blastwave has swept up $\sim 15-40 \mathrm{M}_{\odot}$ of RSG wind (Lee et al., 2010). Estimates of a high swept up mass points to a massive progenitor, with an initial mass of $20-35 \mathrm{M}_{\odot}$.

In general, the range of progenitor models which lead to core collapse supernova remnants is much larger than one sees in the case of Type Ia SNRs. This is reflected in the morphological and compositional diversity of CC SNR we observe in the Galaxy. Ranges in mass loss rates and wind speeds, combined with the initial mass of the star (which will influence the final abundances), as well as evolutionary changes that the progenitor may go through (such as a Wolf-Rayet phase), not to mention the effects that a binary companion can have on the progenitor's evolution, leads to larger parameter space to explore when comparing CC SNR to their progenitors. As seen in Lee et al. (2010) and others, examples exist where broad statements about the progenitor can be made, but few objects allow for the same types of detailed analyses that we find in Ia studies. The exceptions are the Galac- 
tic SNR Cassiopeia A (Cas A), and the youngest know remnant, SN 1987A, in the Large Magallenic Cloud.

Cas A, shown in Figure 5(left), may represent the most well studied of the Galactic SNR. At an age of $330 \mathrm{yr}$ (Thorstensen et al., 2001), it has a morphology which is consistent with that of a SNR interacting with a RSG wind (Chevalier \& Oishi. 2003). Lee et al. (2014); Hwang \& Laming (2012) undertook detailed studies of the mass-loss history of the Cas A progenitor, and estimate that the progenitor star shed $\gtrsim 6 \mathrm{M}_{\odot}$ of material prior to core-collapse. Combined with the estimated mass of the neutron star and ejecta, the progenitor zero-age main sequence mass is $\approx 12 \mathrm{M}_{\odot}$.
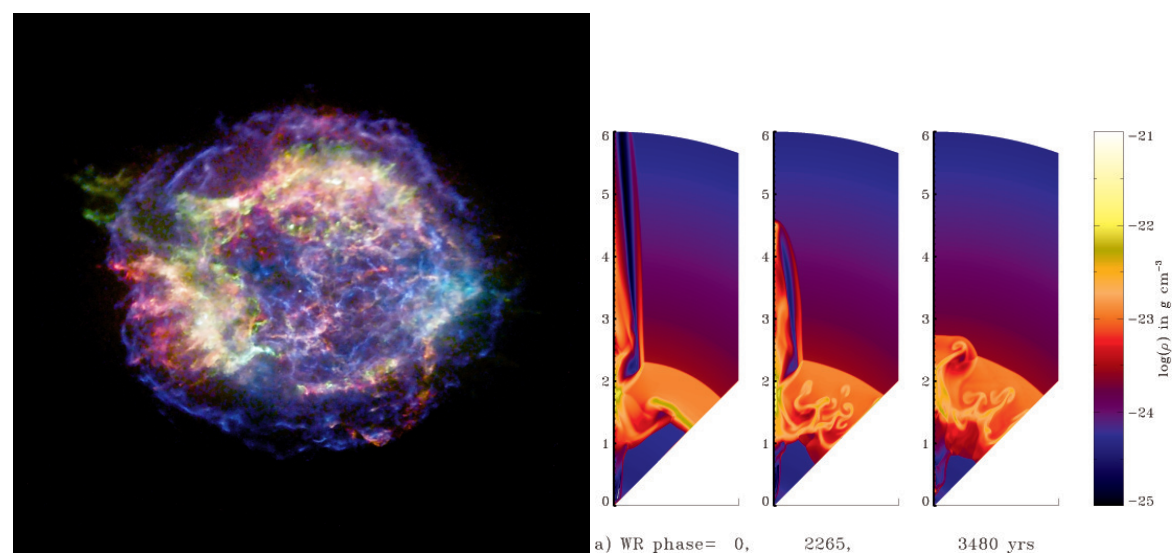

Fig. 5 Left: Cas A in X-rays. The three color image shows $0.5-1.5 \mathrm{keV}$ in red, $1.5-3.0 \mathrm{keV}$ in green, and 4.2-6.0 keV in green. The neutron star is clearly visible near the center. Right: Density of the supernova remnant at a time of $330 \mathrm{yr}$ after explosion. The left panel shows the remnant that results from the evolution of ejecta in a CSM modified by a pure RSG wind, with the middle and righthand panels showing the case where the Wolf-Rayet phase lasted for 2265 and 3480 years, respectively. The forward shock of the remnant in all three cases is located at a distance of $\sim 2.4 \mathrm{pc}$, while the reverse shock is located increasingly farther inward for longer WR phases Details of the models may be found in Schure et al. (2008). (Righthand figure reproduced with the permission of the authors; Schure et al. 2008. Figure 4, pp 403).

The light echo spectra of the Cas A SN obtained by Krause et al. (2008); Rest et al. (2011) suggest a Type IIb origin, similar to SN 1993J, implying a red supergiant origin. The analysis by Lee et al. (2014) suggest that the Cas A progenitor lost much of its mass through the RSG wind, compatible with the IIb SN type, though this is at odds with the mass-loss rates adopted for single RSG stars (Woosley et al. 2002). Solutions to the high amount of mass lost from Cas A include episodic mass loss through pulsational instabilities in the RSG phase Yoon \& Cantiello (2010), or enhanced mass loss through the interaction of a binary companion (Young et al. 2006).

Another possibility is that the Cas A progenitor went through a short Wolf-Rayet phase prior to core collapse. The fast wind from a Wolf-Rayet progenitor would clear out a small cavity, much like is required in Kepler's SNR, and would imprint 
itself on the dynamics and X-ray emission of the remnant. Hwang \& Laming (2012) required such a cavity in order to match the observed X-ray emission. Schure et al. (2008) modeled the evolution of the Cas A jet in a cavity, and found that if a Wolf-Rayet phase existed, it would be rather short ( $\sim$ a few thousand years; Fig. 5 . right).

Besides Cas A, perhaps the most well studied supernova remnant is that of SN 1987A, a recent review of which can be found in McCray \& Fransson (2016). SN 1987A is the closest example of a remnant where the progenitor is known through pre-explosion imaging. However, supernovae are now regularly identified with their progenitors through the use of pre-explosion images. Smartt (2015) notes 18 detections of supernova progenitors, with 27 additional upper limits - a large number of the sample of detected supernova progenitors result in SN IIP, IIL, or IIb. This is consistent with Chevalier (2005), who associated many Galactic SNR with Type II SNe, resulting from RSG progenitors of various masses. Only one or possibly two Galactic SNR have been firmly associated with SNe Type Ib/c (and hence with a Wolf-Rayet progenitor) - W49B as a possible gamma-ray burst remnant (Lopez et al., 2013), and RX J1713-3946 (Katsuda et al., 2015b).

\section{SNR Bulk Properties}

As discussed in the previous sections, typing individual supernova remnants remains challenging. Recently. Yamaguchi et al. (2014) presented a method of typing SNRs based on the $\mathrm{Fe}-\mathrm{K} \alpha$ line centroid and luminosity. Since $\mathrm{Fe}$ is produced in the center of the progenitor during the explosion, heating of $\mathrm{Fe}$ can be delayed, resulting in ionization states lower than $\mathrm{He}$-like $\left(\mathrm{Fe}^{24+}\right)$ in young and middle-aged SNRs. The ionization state affects the Fe-K line centroid, which can be measured with high precision with satellites such as Chandra, XMM-Newton, or Suzaku. In brief, they found a correlation between supernova type, and the Fe-K line centroid: the line centroid for Type Ia SNRs are generally lower $(<6550 \mathrm{eV})$ than those found in CCSNRs. Perhaps more importantly, when they computed synthetic line centroids from models for Type Ia ejecta interacting with a uniform medium, the models predict bulk properties in line with the observations.

Patnaude et al. (2015) extended this work to study core collapse SNR progenitors. While Yamaguchi et al. (2014) focussed on Type Ia SNR in an unmodified circumstellar environment, a key question, as demonstrated in previous sections, is the nature of the circumstellar environment around massive progenitors. Red and Yellow supergiants (R/YSGs) expel several solar masses of material over their lifetimes, with mass loss rates $\approx 10^{-5} \mathrm{M}_{\odot} \mathrm{yr}^{-1}$. Wolf-Rayet stars, thought to be the progenitors of the $\mathrm{Ib} / \mathrm{c}$ subset of core collapse supernovae, expel mass at similar rates, but with wind velocities two orders of magnitude higher.

In addition to steady line-driven mass loss, episodic mass loss may also be relevant. Patnaude et al. (2015) modeled the circumstellar environments of core collapse progenitors for a range of mass loss rates and wind velocities, but did not consider 

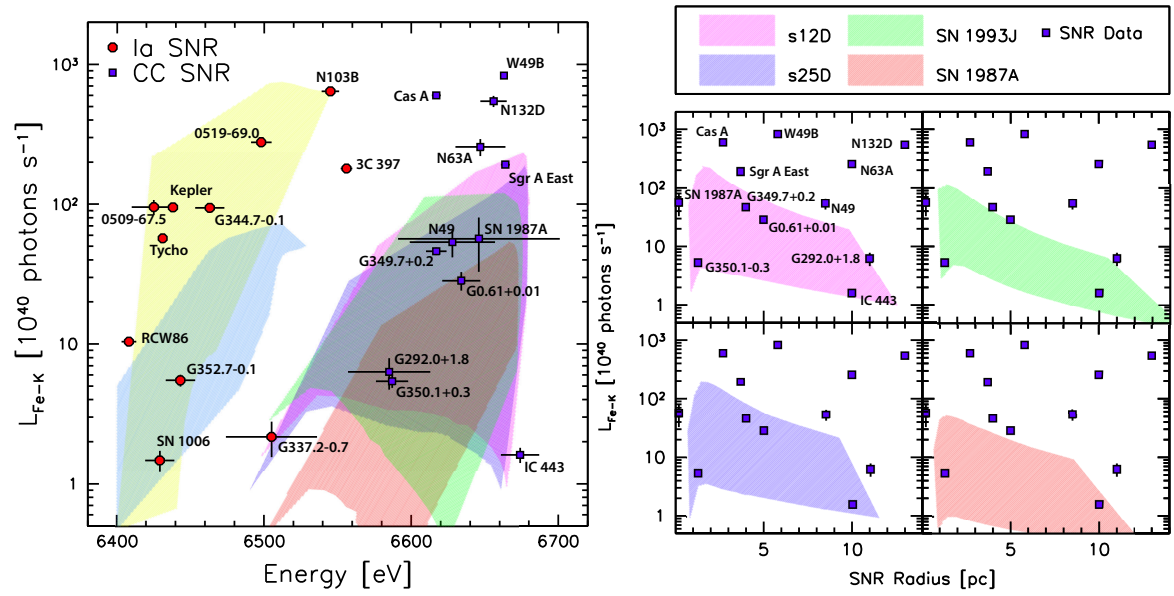

Fig. 6 Left: Fe-K line luminosity vs. centroid energy for Galactic and Magallenic Cloud Ia and core collapse supernova remnants measured by Yamaguchi et al. (2014). The transparent shaded regions correspond to models for either Ia or CCSNe ejecta, with yellow and light blue corresponding to the Ia models DDTa and DDTg, and red, green, magenta, and dark blue corresponding to CCSNe models SN 1987A, SN 1993J, and a 12 and $25 \mathrm{M}_{\odot}$ solar metallicity progenitor, respectively. The data are from Yamaguchi et al. (2014). Right: Fe-K line luminosity as a function of radius for core collapse supernova remnant measurements and models (Figures reproduced with the permission of the authors; Patnaude et al. 2015.

the effects that episodic mass loss would have on their results. In Figure 6(left), we reproduce the results from Patnaude et al. (2015). As seen in the left hand panel, there is a clear bifurcation between Type Ia and CCSNe ejecta models, consistent with the observed properties of the remnants. In particular, the CCSNe models consistently produce higher Fe-K line centroids. Patnaude et al. (2015) attribute this to the blastwave interacting with a great deal of material deposited by the stellar wind around the progenitor.

While these models capture the general spectral characteristics of the data, when correlating modeled radii to line luminosity, an apparent disconnect is seen between the modeled line luminosities as a function of radius, as compared to the data. This is shown in Figure 6(right), where the models underestimate the $\mathrm{Fe}-\mathrm{K}$ line luminosity compared to the data.

In the case of CCSNe, the Fe-K line centroid is already close to the collisional ionization equilibrium value. Modest increases in the progenitor mass loss rate will have a small effect on the ionization, while providing a boost to the line luminosity, since the X-ray emission scales like the square of the density. Increases in the mass loss rate, if non-steady, would not affect the radius, as the interaction timescale between the blast wave and the additional circumstellar material would be short, compared to the overall age of the remnant.

There is now growing evidence that core collapse progenitors undergo some sort of episodic mass loss prior to core collapse. The optical light curves of several Type IIn supernovae, including SN 2005ip, SN 2005kj, and SN 2006aa, all suggest 
episodic mass loss prior to core collapse (Moriya et al., 2014), and a mass loss event from the progenitor of SN 2009ip was sufficiently luminous that it was misclassified as a SN, though the progenitor did finally explode in 2012. The mechanism for the episodic mass loss is not currently understood, but the mass ejection may be related to wave-driven mass loss during core neon and oxygen burning (Shiode \& Quataert. 2014), or possibly unsteady nuclear burning in the envelope (Smith 2014).

The mass loss history, steady or otherwise, will imprint itself on the X-ray emission from the SNR (Patnaude et al. 2015). As an example of this, Moriya (2012) investigated the progenitors for so-called recombining supernova remnants, remnants where the ion temperature is sufficiently higher than the electron temperature, and found that a plausible explanation exists for them if their progenitors lost a sufficient amount of mass that was subsequently deposited near the progenitor, just prior to core-collapse. They argued that the progenitors of these remnants are likely red supergiants, and are likely not associated with SNe IIn.

\section{Conclusions}

In this chapter we have reviewed several properties of supernova remnants which provide links back to their progenitor systems. X-ray observations of supernova remnants place constraints on the metallicity of the progenitor and measuring the explosion energetics, either through proper motion analyses or inferred from spectral measurements can provide constraints on the explosion energetics, as well as the density of the circumstellar medium. These parameters relate directly back to the evolution of the progenitor.

In the case of Type Ia SNRs, measuring the abundances of neutron-rich material places constraints on the central density of the white dwarf progenitor. Inferring a high central density points to a $\mathrm{M}_{\mathrm{Ch}}$ explosion and likely a single-degenerate progenitor system. Likewise, joint spectral/dynamical fits to objects such as Kepler's SNR point to complicated evolutionary histories for the progenitor, where an AGB wind is required from the progenitor system, but only if it has a cavity excised out of it prior to the supernova event. A similar result has been suggested in Tycho (Chiotellis et al. 2013, Slane et al. 2014).

For progenitors od core-collapse SNR, the situation is more complicated. CCSNe progenitors exhibit a larger mass range, with order of magnitude variations in mass loss rates and progenitor wind velocities. Except for the notable cases of SN 1987A and Cas A, only broad statements about the SNR progenitor may be made at this time. However, when one begins to step back from individual remnants, trends in SNR bulk properties begin to emerge. Recent results by Yamaguchi et al. (2014) show a clear bifurcation in line centroids between SNR Ia and core-collapse SNRs. These results are consistent with model predictions.

While models for SNR progenitors do generally agree with observed bulk properties of SNR, there are still inconsistencies. Nonetheless, these issues may be addressed by stellar evolution models which better define the end stages of the pro- 
genitor, including such affects as episodic mass loss and non-steady nuclear burning. Both mechanisms alter the circumstellar environment in a way that is readily observable in remnants, hundreds of years after the supernova event.

\section{Acknowledgments}

The authors wish to thank the editorial staff for their steadfast patience during the preparation of this manuscript. D. J. P. acknowledges support from NASA contract NAS8-03060 and NASA/TM6-17003X. C. B. acknowledges partial support from grants NSF/AST-1412980 and NASA/NNX15AM03G-S01.

\section{Cross References}

- Supernova Remnant Cassiopeia A

- Observational Classification of Supernovae

- Dynamical Evolution and Radiative Processes in Supernova Remnants

- Supernova remnant from SN 1987A

\section{References}

Badenes, C., Bravo, E., Borkowski, K. J., \& Domínguez, I. 2003, “Thermal X-Ray Emission from Shocked Ejecta in Type Ia Supernova Remnants: Prospects for Explosion Mechanism Identification,” ApJ, 593, 358

Badenes, C., Borkowski, K. J., \& Bravo, E. 2005, "Thermal X-Ray Emission from Shocked Ejecta in Type Ia Supernova Remnants. II. Parameters Affecting the Spectrum," ApJ, 624, 198

Badenes, C., Borkowski, K. J., Hughes, J. P., Hwang, U., \& Bravo, E. 2006, "Constraints on the Physics of Type Ia Supernovae from the X-Ray Spectrum of the Tycho Supernova Remnant," ApJ, 645, 1373

Badenes, C., Hughes, J. P., Bravo, E., \& Langer, N. 2007, "Are the Models for Type Ia Supernova Progenitors Consistent with the Properties of Supernova Remnants?," ApJ, 662, 472

Badenes, C., Harris, J., Zaritsky, D., \& Prieto, J. L. 2009, "The Stellar Ancestry of Supernovae in the Magellanic Clouds. I. The Most Recent Supernovae in the Large Magellanic Cloud," ApJ, 700, 727

Badenes, C. 2010, "X-ray studies of supernova remnants: A different view of supernova explosions," Proceedings of the National Academy of Science, 107, 7141

Badenes, C., \& Maoz, D. 2012, "The Merger Rate of Binary White Dwarfs in the Galactic Disk," ApJ, 749, L11 
Borkowski, K. J., Lyerly, W. J., \& Reynolds, S. P. 2001, "Supernova Remnants in the Sedov Expansion Phase: Thermal X-Ray Emission," ApJ, 548, 820

Borkowski, K. J., Reynolds, S. P., Green, D. A., et al. 2014, "Nonuniform Expansion of the Youngest Galactic Supernova Remnant G1.9+0.3," ApJ, 790, L18

Brachwitz, F., Dean, D. J., Hix, W. R., et al. 2000, "The Role of Electron Captures in Chandrasekhar-Mass Models for Type IA Supernovae," ApJ, 536, 934

Chevalier, R. A., \& Oishi, J. 2003, "Cassiopeia A and Its Clumpy Presupernova Wind," ApJ, 593, L23

Chevalier, R. A. 2005, "Young Core-Collapse Supernova Remnants and Their Supernovae," ApJ, 619, 839

Chiotellis, A., Schure, K. M., \& Vink, J. 2012, "The imprint of a symbiotic binary progenitor on the properties of Kepler's supernova remnant," A\&A, 537, A139

Chiotellis, A., Kosenko, D., Schure, K. M., Vink, J., \& Kaastra, J. S. 2013, "Modelling the interaction of thermonuclear supernova remnants with circumstellar structures: the case of Tycho's supernova remnant," MNRAS, 435, 1659

Chomiuk, L., Soderberg, A. M., Chevalier, R. A., et al. 2016, "A Deep Search for Prompt Radio Emission from Thermonuclear Supernovae with the Very Large Array," ApJ, 821, 119

DeLaney, T., \& Rudnick, L. 2003, “The First Measurement of Cassiopeia A's Forward Shock Expansion Rate," ApJ, 589, 818

Dessart, L., Blondin, S., Hillier, D. J., \& Khokhlov, A. 2014, "Constraints on the explosion mechanism and progenitors of Type Ia supernovae," MNRAS, 441, 532

Edwards, Z. I., Pagnotta, A., \& Schaefer, B. E. 2012, "The Progenitor of the Type Ia Supernova that Created SNR 0519-69.0 in the Large Magellanic Cloud," ApJ, 747, L19

Ferrière, K. M. 2001, “The interstellar environment of our galaxy," Reviews of Modern Physics, 73, 1031

Filippenko, A. V. 1997, “Optical Spectra of Supernovae,” ARA\&A, 35, 309

Hachisu, I., Kato, M., \& Nomoto, K. 1996, "A New Model for Progenitor Systems of Type IA Supernovae," ApJ, 470, L97

Hillebrandt, W., Kromer, M., Röpke, F. K., \& Ruiter, A. J. 2013, "Towards an understanding of Type Ia supernovae from a synthesis of theory and observations," Frontiers of Physics, 8, 116

Hwang, U., \& Laming, J. M. 2009, "The Circumstellar Medium of Cassiopeia a Inferred from the Outer Ejecta Knot Properties," ApJ, 703, 883

Hwang, U., \& Laming, J. M. 2012, "A Chandra X-Ray Survey of Ejecta in the Cassiopeia A Supernova Remnant," ApJ, 746, 130

Jennings, Z. G., Williams, B. F., Murphy, J. W., et al. 2014, “The Supernova Progenitor Mass Distributions of M31 and M33: Further Evidence for an Upper Mass Limit," ApJ, 795, 170

Katsuda, S., Mori, K., Maeda, K., et al. 2015, "Kepler's Supernova: An Overluminous Type Ia Event Interacting with a Massive Circumstellar Medium at a Very Late Phase," ApJ, 808, 49

Katsuda, S., Acero, F., Tominaga, N., et al. 2015, ApJ, 814, 29 
Kerzendorf, W. E., Childress, M., Scharwächter, J., Do, T., \& Schmidt, B. P. 2014, "A Reconnaissance of the Possible Donor Stars to the Kepler Supernova," ApJ, 782,27

Koo, B.-C., \& McKee, C. F. 1992, "Dynamics of wind bubbles and superbubbles. I - Slow winds and fast winds. II - Analytic theory," ApJ, 388, 93

Krause, O., Birkmann, S. M., Usuda, T., et al. 2008, "The Cassiopeia A Supernova Was of Type IIb," Science, 320, 1195

bibitem[Kerzendorf et al.(2014)]kerzendorf14 Kerzendorf, W. E., Childress, M., Scharwächter, J., Do, T., \& Schmidt, B. P. 2014, "A Reconnaissance of the Possible Donor Stars to the Kepler Supernova," ApJ, 782, 27

Lee, J.-J., Park, S., Hughes, J. P., et al. 2010, "The Outer Shock of the Oxygen-Rich Supernova Remnant G292.0+1.8: Evidence for the Interaction with the Stellar Winds from Its Massive Progenitor," ApJ, 711, 861

Lee, J.-J., Park, S., Hughes, J. P., \& Slane, P. O. 2014, "X-Ray Observation of the Shocked Red Supergiant Wind of Cassiopeia A," ApJ, 789, 7

Lopez, L. A., Ramirez-Ruiz, E., Castro, D., \& Pearson, S. 2013, "The Galactic Supernova Remnant W49B Likely Originates from a Jet-driven, Core-collapse Explosion," ApJ, 764, 50

Maggi, P., Haberl, F., Kavanagh, P. J., et al. 2016, "The population of X-ray supernova remnants in the Large Magellanic Cloud," A\&A, 585, A162

Maoz, D., Mannucci, F., \& Nelemans, G. 2014, "Observational Clues to the Progenitors of Type Ia Supernovae," ARA\&A, 52, 107

Margutti, R., Parrent, J., Kamble, A., et al. 2014, "No X-Rays from the Very Nearby Type Ia SN 2014J: Constraints on Its Environment," ApJ, 790, 52

Maund, J. R., Smartt, S. J., Kudritzki, R. P., Podsiadlowski, P., \& Gilmore, G. F. 2004, "The massive binary companion star to the progenitor of supernova 1993J," Nature, 427, 129

McCray, R., \& Fransson, C. 2016, “The Remnant of Supernova 1987A," ARA\&A, 54,19

Moriya, T. J. 2012, "Progenitors of Recombining Supernova Remnants," ApJ, 750, L13

Moriya, T. J., Maeda, K., Taddia, F., et al. 2014, "Mass-loss histories of Type IIn supernova progenitors within decades before their explosion," MNRAS, 439, 2917

Park, S., Badenes, C., Mori, K., et al. 2013, "A Super-solar Metallicity for the Progenitor of Kepler's Supernova," ApJ, 767, L10

Patnaude, D. J., \& Fesen, R. A. 2009, "Proper Motions and Brightness Variations of Nonthermal X-ray Filaments in the Cassiopeia A Supernova Remnant", ApJ, 697,535

Patnaude, D. J., Badenes, C., Park, S., \& Laming, J. M. 2012, “The Origin of Kepler's Supernova Remnant," ApJ, 756, 6

Patnaude, D. J., Lee, S.-H., Slane, P. O., et al. 2015, "Are Models for Core-collapse Supernova Progenitors Consistent with the Properties of Supernova Remnants?," ApJ, 803, 101 
Pérez-Torres, M. A., Lundqvist, P., Beswick, R. J., et al. 2014, "Constraints on the Progenitor System and the Environs of SN 2014J from Deep Radio Observations," ApJ, 792, 38

Ponder, K. A., Wood-Vasey, W. M., \& Zentner, A. R. 2016, "Incorporating Astrophysical Systematics into a Generalized Likelihood for Cosmology with Type Ia Supernovae," ApJ, 825, 35

Raymond, J. C., Korreck, K. E., Sedlacek, Q. C., et al. 2007, "The Preshock Gas of SN 1006 from Hubble Space Telescope Advanced Camera for Surveys Observations," ApJ, 659, 1257

Rest, A., Welch, D. L., Suntzeff, N. B., et al. 2008, "Scattered-Light Echoes from the Historical Galactic Supernovae Cassiopeia A and Tycho (SN 1572)," ApJ, 681, L81

Rest, A., Foley, R. J., Sinnott, B., et al. 2011, "Direct Confirmation of the Asymmetry of the Cas A Supernova with Light Echoes," ApJ, 732, 3

Saio, H., \& Nomoto, K. 1985, "Evolution of a merging pair of C+O white dwarfs to form a single neutron star," A\&A, 150, L21

Schaefer, B. E., \& Pagnotta, A. 2012, "An absence of ex-companion stars in the type Ia supernova remnant SNR 0509-67.5," Nature, 481, 164

Schure, K. M., Vink, J., García-Segura, G., \& Achterberg, A. 2008, "Jets as Diagnostics of the Circumstellar Medium and the Explosion Energetics of Supernovae: The Case of Cassiopeia A," ApJ, 686, 399-407

Shen, K. J., Guillochon, J., \& Foley, R. J. 2013, "Circumstellar Absorption in Double Detonation Type Ia Supernovae," ApJ, 770, L35

Shen, K. J., \& Bildsten, L. 2007, "Thermally Stable Nuclear Burning on Accreting White Dwarfs," ApJ, 660, 1444

Shiode, J. H., \& Quataert, E. 2014, "Setting the Stage for Circumstellar Interaction in Core-Collapse Supernovae. II. Wave-driven Mass Loss in Supernova Progenitors," ApJ, 780, 96

Slane, P., Lee, S.-H., Ellison, D. C., et al. 2014, "A CR-hydro-NEI Model of the Structure and Broadband Emission from Tycho's Supernova Remnant "' ApJ, 783,33

Smartt, S. J., Maund, J. R., Gilmore, G. F., et al. 2003, "Mass limits for the progenitor star of supernova 2001du and other Type II-P supernovae," MNRAS, 343, 735

Smartt, S. J. 2015, "Observational Constraints on the Progenitors of Core-Collapse Supernovae: The Case for Missing High-Mass Stars," Publications of the Astronomical Society of Australia, 32, e016

Smith, N. 2014, "Mass Loss: Its Effect on the Evolution and Fate of High-Mass Stars," ARA\&A, 52, 487

Thorstensen, J. R., Fesen, R. A., \& van den Bergh, S. 2001, "The Expansion Center and Dynamical Age of the Galactic Supernova Remnant Cassiopeia A," AJ, 122, 297

van Kerkwijk, M. H., Chang, P., \& Justham, S. 2010, "Sub-Chandrasekhar White Dwarf Mergers as the Progenitors of Type Ia Supernovae," ApJ, 722, L157 
Van Dyk, S. D., Garnavich, P. M., Filippenko, A. V., et al. 2002, "The Progenitor of Supernova 1993J Revisited," PASP, 114, 1322

Van Dyk, S. D., Li, W., \& Filippenko, A. V. 2003, "On the Progenitor of the Type II-Plateau Supernova 2003gd in M74," PASP, 115, 1289

Vink, J. 2012, "Supernova remnants: the X-ray perspective," A\&A Rev., 20, 49

Wang, B., \& Han, Z. 2012, "Progenitors of type Ia supernovae," New A Rev., 56, 122

Williams, B. J., Blair, W. P., Blondin, J. M., et al. 2011, "RCW 86: A Type Ia Supernova in a Wind-blown Bubble," ApJ, 741, 96

Williams, B. F., Peterson, S., Murphy, J., et al. 2014, "Constraints for the Progenitor Masses of 17 Historic Core-collapse Supernovae," ApJ, 791, 105

Woosley, S. E., Heger, A., \& Weaver, T. A. 2002, "The evolution and explosion of massive stars," Reviews of Modern Physics, 74, 1015

Yamaguchi, H., Badenes, C., Petre, R., et al. 2014, "Discriminating the Progenitor Type of Supernova Remnants with Iron K-shell Emission,” ApJ, 785, L27

Yamaguchi, H., Badenes, C., Foster, A. R., et al. 2015, "A Chandrasekhar Mass Progenitor for the Type Ia Supernova Remnant 3C 397 from the Enhanced Abundances of Nickel and Manganese," ApJ, 801, L31

Yang, X. J., Tsunemi, H., Lu, F. J., et al. 2013, "Cr-K Emission Line as a Constraint on the Progenitor Properties of Supernova Remnants," ApJ, 766, 44

Yoon, S.-C., \& Cantiello, M. 2010, "Evolution of Massive Stars with Pulsationdriven Superwinds During the Red Supergiant Phase," ApJ, 717, L62

Young, P. A., Fryer, C. L., Hungerford, A., et al. 2006, "Constraints on the Progenitor of Cassiopeia A," ApJ, 640, 891

Zhou, P., Chen, Y., Zhang, Z.-Y., et al. 2016, "Expanding Molecular Bubble Surrounding Tycho's Supernova Remnant (SN 1572) Observed with the IRAM 30m Telescope: Evidence for a Single-degenerate Progenitor,” ApJ, 826, 34 